\title{
Quantum-mechanical simulations of low-frequency current excitation during ionization of many-electron atoms by intense laser pulses
}

\author{
$\underline{\text { A. A. Silaev }}{ }^{1,2}$, A. A. Romanov ${ }^{1,2}$, N. V. Vvedenskii ${ }^{1,2}$ \\ ${ }^{1}$ Institute of Applied Physics, Russian Academy of Sciences, Nizhny Novgorod, Russia, silaev@appl.sci-nnov.ru \\ ${ }^{2}$ University of Nizhny Novgorod, Nizhny Novgorod, Russia
}

Great progress in computer technology opens up the possibility for $a b$ initio numerical study of various ionization-stimulated phenomena arising from the interaction of atoms and molecules with intense laser pulses. These include the excitation of a lowfrequency current (with frequencies much lower than the optical one) in a plasma created by a laser pulse. The interest to this phenomenon is associated with the possibility of its use for the generation of coherent and broadband radiation in the terahertz and midinfrared ranges and possibility of tuning the spectrum of generated radiation [1-11]. The low-frequency current density is excited effitiently under the asymmetry of ionizing pulses [1-10] or ionized molecules [11]. Among the various methods of generating low-frequency current, the most wellknown one is based on the use of two-color ionizing laser pulses [1-8]. The low-frequency current is generated at some combination frequency of twocomponent laser pulse for a whole set of ratios between component frequencies [5-8].

The efficient excitation of high-power terahertz and mid-infrared radiation is associated with the need to use of high-intensity laser pulses, which produce fast ionization of a gas medium and accelerate the freed electrons up to high energies $[2,5,7,9,10]$. The action of such intense pulses on atoms or molecules (most of which are multielectron quantum systems) may be accompanied by a dynamic response of various electron orbitals, which was not taken into account in previous similar studies. This response can significantly influence the magnitude and waveform of the low-frequency current.

This paper is devoted to direct numerical simulations of ionization-induced excitation of lowfrequency electron current density in multielectron atoms of noble gases. Our approach is based on the time-dependent density functional theory (TDDFT), which has recently been increasingly used in atomic, molecular, and strong-field physics [12]. The basis of this approach is the system of time-dependent KohnSham (TDKS) equations, which takes into account the interaction of electrons with the atomic nucleus and the external electric field of the laser pulse, as well as the electron-electron interaction. In the atomic system of units (in which $|e|=\hbar=m_{\mathrm{e}}=1$, where $\hbar$ is the reduced Planck constant, $e=-|e|$ is the charge and $m_{\mathrm{e}}$ is the mass of the electron) these equations are:

$$
\begin{aligned}
& i \frac{\partial}{\partial t} \psi_{n}(\mathbf{r}, t)=\hat{H} \psi_{n}(\mathbf{r}, t), \quad n=1, \ldots, N, \\
& \hat{H}=-\frac{1}{2} \nabla^{2}-\frac{Z}{r}+\mathbf{r E}(t)+V_{\mathrm{ee}}[\rho(\mathbf{r}, t)] .
\end{aligned}
$$

Here, $\psi_{n}$ is the wave function of the $n$-th TDKS orbital, $Z$ is the nuclear charge, $N$ is the number of electrons, $\rho(\mathbf{r}, \mathrm{t})$ is the electron density, and $V_{e e}[\rho(\mathbf{r}, \mathrm{t})]$ is the potential of electron-electron interaction. The initial Kohn-Sham orbitals correspond to electronic configuration of the unperturbed atom. For considered noble-gas atoms, the atomic orbitals are initially occupied by the pairs of electrons with opposite spin. Due to the weak influence of the magnetic field, the zero spin polarization is conserved during interaction with the laser pulse. The electron density is related to TDKS orbitals by equality

$$
\rho(\mathbf{r}, t)=\sum_{n=1}^{N}\left|\psi_{n}(\mathbf{r}, t)\right|^{2} .
$$

The electron-electron interaction potential consists of the Hartree potential $V_{\mathrm{H}}[\rho(\mathbf{r}, t)]=\int d^{3} r^{\prime} \rho\left(\mathbf{r}^{\prime}\right) /\left|\mathbf{r}-\mathbf{r}^{\prime}\right|$, describing the electron repulsion in the framework of the mean field and the exchange-correlation potential for which the spin unpolarized form of LB94 approximation [12] is used.

Using TDKS orbitals and Ehrenfest's theorem we find the time-dependent dipole acceleration of the atomic system:

$$
\mathbf{a}(t)=-N \mathbf{E}(t)-\int d^{3} r \frac{Z \mathbf{r}}{r^{3}} \rho(\mathbf{r}, t) .
$$

The obtained dipole acceleration is proportional to the time derivative of macroscopic electron current density,

$$
\frac{\partial \mathbf{j}(t)}{\partial t}=-N_{m} \mathbf{a}(t)
$$

where $N_{m}$ is the gas density before the start of the ionization process.

Based on the developed computer code for solving the 3D TDKS, we calculate the current density $\mathbf{j}(t)$ excited by two-color laser pulses containing the main and second harmonics in a wide range of intensities and durations of the laser-pulse components. The found electron current density $\mathbf{j}(t)$ has a very wide frequency spectrum. Besides the lowfrequency part, it contains a large component at the frequency of the ionizing laser pulse and its low-order harmonics. Also it contains a wide plateau ending with a pronounced cutoff in the frequency region, which corresponds to the extreme UV or soft-X-ray radiation [13]. We extract the low-frequency component from the full current density and study differences in its excitation for different noble-gas atoms. The results obtained on the basis of TDDFT are compared with the calculations of the singleactive electron (SAE) approximation. In the framework of SAE approximation all orbitals except highest-occupied orbital are "frozen", and the field of 
the parent ion is described by a static potential well. It is shown that taking into account the dynamics of all the electrons can lead to a change in the optimal relative phase between the components of the twocolor laser pulse and in the corresponding maximum low-frequency current. Based on the results obtained, the range of applicability of SAE approximation is determined.

This work was supported by the Russian Foundation for Basic Research (Grant Nos. 16-3260200, 18-02-01150, 18-32-00951) and the Russian Science Foundation (numerical calculations, Grant No. 18-72-00192).

\section{References}

1. Clough B., Dai J., Zhang, X.-C. Laser air photonics: beyond the terahertz gap // Mater. Today. 2012. V. 15, No.1-2. P. 50-58.

2. Vvedenskii, N. V., et al. Two-color laser-plasma generation of terahertz radiation using a frequency-tunable half harmonic of a femtosecond pulse // Phys. Rev. Lett. 2014. 112, No.5. P. 055004.

3. Balčiūnas, T. D. et al. CEP-stable tunable THzemission originating from laser-waveform-controlled subcycle plasma-electron bursts // Opt. Express. 2015. V. 23, No. 12. P. 15278-15289.

4. Theberge, F. et al. Generation of tunable and broadband far-infrared laser pulses during two-color filamentation // Phys. Rev. A 2010. V. 81, No.3. P. 033821.
5. Kostin, V. A., Laryushin, I. D., Silaev, A. A., Vvedenskii, $N$. $V$. Ionization-induced multiwave mixing: terahertz generation with two-color laser pulses of various frequency ratios // Phys. Rev. Lett. 2016. V. 117, No. 3. P. 035003.

6. Zhang, L.-L. et al. Observation of terahertz radiation via the two-color laser scheme with uncommon frequency ratios // Phys. Rev. Lett. 2017. V.119, No. 23. P. 235001.

7. Silaev, A. A., Kostin, V. A., Laryushin, I. D., Vvedenskii, $N$. $V$. Ionization mechanism of the generation of tunable ultrashort pulses in the mid-infrared range // JETP Letters. 2018. V. 107, No. 3. P.151-156.

8. Kostin V.A., Vvedenskii N.V. Generation of Few- and Subcycle Radiation in Midinfrared-to-Deep-Ultraviolet Range During Plasma Production by Multicolor Femtosecond Pulses // Phys. Rev. Lett. V. 120, No. 6. P. 065002.

9. Silaev, A.A., Vvedenskii, N. V. Residual-current excitation in plasmas produced by few-cycle laser pulses // Phys. Rev. Lett. 2009. V. 102, No. 11. P. 115005.

10. Silaev, A. A., Vvedenskii N. V. Analytical description of generation of the residual current density in the plasma produced by a few-cycle laser pulse // Phys. Plasmas. 2015. V. 22, No. 5. P. 053103.

11. Alexandrov, L. N., Emelin, M. Yu., Ryabikin M. Yu. Unidirectional current excitation in tunneling ionization of asymmetric molecules // Phys. Rev. A. 2013. V. 87, No. 1. P. 013414.

12. Ullrich, C. A. Time-dependent density-functional theory: concepts and applications // Oxford University Press. 2012.

13. Krausz, F., Ivanov, M. Attosecond physics // Rev. Mod. Phys. 2009. V. 81, No. 1. P. 163. 